\title{
BIOMECHANICAL PROPERTIES OF CORNEAL EXTRACELLULAR MATRIX USING EXPERIMENTAL AND COMPUTATIONAL METHODS
}

\author{
Hamed Hatami-Marbini ${ }^{1}$ \\ ${ }^{1}$ Department of Mechanical \& Industrial Engineering, \\ University of Illinois at Chicago, Chicago, Illinois 60607 \\ e-mail: hatami@uic.edu
}

Keywords: cornea, soft tissue, computational method, experiments, mechanical behavior, hydration.

\begin{abstract}
The cornea is a transparent soft tissue at the front of the eye and is mainly responsible for refracting the incoming light and protecting the internal contents of the eye. Although the cornea is composed of different components, it is well-known that its biomechanical properties are due to the structure and function of its extracellular matrix, called stroma. The stroma is composed of regularly arranged collagen fibers embedded in a hydrated proteoglycans matrix. From a mechanics viewpoint, the stroma can be considered as a composite structure in which the collagen fibrils and the hydrated matrix take tensile and compressive forces, respectively.

In this work, experimental and computational studies conducted to fully characterize the mechanical properties of the cornea in compression and tension. The unconfined compression experiments were conducted on corneal disks while uniaxial tensile tests were performed on corneal rectangular strips. A transversely isotropic constitutive model was used to numerically analyze the experimental measurements and determine the material constants of the cornea. It is shown that the material properties of the cornea in tension and compression are hydration dependent. Therefore, careful attention must be taken in corneal hydration during material characterization experiments.
\end{abstract}




\section{INTRODUCTION}

The cornea is a hydrated soft tissue which transmits the incident light while acting as a resilient barrier to the mechanical forces. From the anterior to posterior, the cornea is composed of epithelial cells, Bowman's zone, stroma, Descemet's membrane, and endothelial cells. The biomechanical properties of the cornea are mainly derived from the stromal layer. The mechanical behavior of corneal stroma can be determined using uniaxial strip extentiometry, inflation tests, indentation tests, and compressive experiments.

The uniaxial tensile tests are done using rectangular corneal strips subjected to tensile loading [1-8]. Because of the well-known limitations of this testing technique [9], it has often been used for comparative studies $[3,6,10]$. Compared to the uniaxial testing technique, inflation tests are able to better represent natural conditions of the cornea as they do not use excised samples [11, 12]. However, the use of pressurization fluid during inflation experiments results in corneal swelling $[8,11]$. This unwanted swelling may affect the precision of experimental measurements. The compressive experiments have also been used to study the mechanical behavior of the cornea in terms of its swelling pressure-thickness relation [13-17]. The findings of the above experimental studies show a wide range of variation in the reported material parameters for the corneal tissue. This variation has been often explained in terms of inherent differences in sample properties and experimental conditions.

The objective of the present manuscript is to show that corneal mechanical properties are hydration dependent and the lack of control on tissue hydration explain the existing discrepancies in the literature. For this purpose, we investigated the effects of hydration on tensile and compressive properties of the cornea [18-22]. We performed unconfined compression experiments and used a transversely isotropic biphasic model to determine corneal material parameters as a function of hydration.

\section{MATERIALS AND METHODS}

\subsection{Sample Preparation}

Porcine and bovine eyes were obtained from a slaughterhouse. At the laboratory, corneoscleral rims were excised from normal looking corneas. The epithelium and endothelium were removed from the corneal surface. A biopsy punch and double blade device were used to prepare corneal disks and corneal strips. The stromal buttons were weighed with $0.1 \mathrm{mg}$ accuracy and their thickness was measured using an ultrasound pachymeter. The stromal buttons were used in unconfined experiments. The corneal strips were divided into the following groups based on their average thicknesses: $0.3,0.35,0.4,0.5,0.7,0.9$, and $1.1 \mathrm{~mm}$. In order to alter the thickness (hydration) of the strips, all specimens were air-dried in a desiccator and were then immersed in an ionic solution until the desired thickness of one of the groups was obtained. In order to obtain the hydration of the specimens, the linear hydration-thickness relation, i.e. $H_{w}=5.3 t-0.67$, was used [23]. In hydration-thickness relation, $H_{w}$ is hydration in $\mathrm{mg}$ water / mg dry tissue and $t$ denotes the thickness in $\mathrm{mm}$.

\subsection{Uniaxial Tensile Experiments}

The samples were tested using a custom-built micro tensile device composed of a linear stepper motor, a submersible load cell, and serrated grips. The strips were mounted between serrated grips and glue was used to prevent any possible slippage. Mineral oil was used to fill the chamber in order to prevent any changes in the hydration (thickness) of the specimens. In order to remove possible variations in experimental measurements caused by stress-strain his- 
tory, a preconditioning step was done at the beginning of all experiments [6, 7]. The preconditioning was composed of five loading/unloading cycles and three successive relaxation tests. A tare load of $100 \mathrm{mN}$ was then applied to straighten the samples and determine the initial length of specimens. This initial length was used to obtain the engineering strain. The experiments were conducted using an engineering strain of $5 \%$ and a displacement rate of $2 \mathrm{~mm} / \mathrm{min}$. The stress was calculated from dividing the recorded force by the initial cross-sectional area.

\subsection{Unconfined Compression Experiments}

A DHR-2 Rheometer was used to perform unconfined compression experiments. The chamber of the rheometer was first filled with $\mathrm{NaCl}$ solution and the corneal buttons were placed on the center of the impermeable platen surface. Initially A preload equivalent to a stress of $1.5 \mathrm{KPa}$ was applied in order to create a uniform starting point in all experiments. The equilibrium thickness of corneal disks at this load was taken as the initial thickness for calculating engineering strain. A series of ramp-hold compressive experiments was then performed. The reaction stress was calculated by dividing the recorded force by the initial area of the samples. A constant displacement rate of $0.15 \mu \mathrm{m} / \mathrm{s}$ was used in all experiments.

\subsection{Data Analysis}

The stress-strain curves obtained from uniaxial experiments were represented using the exponential expression $\sigma=A\left(e^{B \varepsilon}-1\right)+\sigma_{0}$ and power-law function $\sigma=\alpha \varepsilon^{\beta}+\sigma_{0}[1,24]$. Here, $\varepsilon$ is the engineering strain, $\sigma$ is the stress, $\sigma_{0}$ is the initial tare stress and A, $B, \alpha$, and $\beta$ are fit parameters.

The unconfined compression experimental measurements at each ramp-and-hold were curve-fitted to a transversely isotropic biphasic model. The compressive stress of a stromal disk with radius $a$ subjected to ramp-hold compressive strain is given by [25],

$$
\sigma(t)=\left\{\begin{array}{c}
E_{z} \dot{\varepsilon}_{0} t+E_{r} \frac{\dot{\varepsilon}_{0} a^{2}}{\kappa_{r} \mathrm{H}} B_{3}\left(\frac{1}{8}-\sum_{n=1}^{\infty} \frac{e^{-x_{n}{ }^{2} \kappa_{r} \mathrm{H}^{-2} t}}{x_{n}^{2}\left(B_{2}^{2} x_{n}^{2}-B_{1}\left(1+v_{r \theta}\right)^{-1}\right)}\right) t \leq t_{0} \\
E_{z} \dot{\varepsilon}_{0} t_{0}-E_{r} \frac{\dot{\varepsilon}_{0} a^{2}}{\kappa_{r} \mathrm{H}} B_{3} \sum_{n=1}^{\infty} \frac{e^{-x_{n}^{2} \kappa_{r} \mathrm{H}^{-2} t}-e^{-x_{n}{ }^{2} \kappa_{r} \mathrm{H}^{-2}\left(t-t_{0}\right)}}{x_{n}^{2}\left(B_{2}{ }^{2} x_{n}{ }^{2}-B_{1}\left(1+v_{r \theta}\right)^{-1}\right)} \quad t \geq t_{0}
\end{array}\right.
$$

where

$$
\begin{aligned}
& B_{1}=1-v_{r \theta}-2 v_{z r}{ }^{2} E_{r} \mathrm{E}_{z}^{-1}, B_{2}=\left(1-v_{z r}{ }^{2} E_{r} \mathrm{E}_{z}^{-1}\right)\left(1+v_{r \theta}\right)^{-1}, \\
& B_{3}=\left(1-2 v_{z r}^{2}\right) B_{2} B_{1}^{-1}, \mathrm{H}=E_{r}\left(1-v_{z r}{ }^{2} E_{r} \mathrm{E}_{z}^{-1}\right)\left(\left(1+v_{r \theta}\right) B_{1}\right)^{-1},
\end{aligned}
$$

$t_{0}$ is the ramp time, $\dot{\varepsilon}_{0}$ is the ramp compressive strain rate, $E_{r}$ is the in-plane Young's modulus, $E_{z}$ is the out-of-plane Young's modulus, $v_{r} \theta$ is the in-plane Poison's ratio, $v_{z r}$ is out-of-plane Poison's ratio, $\kappa_{r}$ is the permeability coefficient and $x_{n}$ 's are the solution of following equation

$$
J_{1}(x)-\frac{1-v_{z r}^{2} E_{r} \mathrm{E}_{z}^{-1}}{1-v_{r \theta}-2 v_{z r}^{2} E_{r} \mathrm{E}_{z}^{-1}} x J_{0}(x)=0,
$$


and $J_{0}$ and $J_{1}$ are the zeroth and first order Bessel functions of the first kind, respectively. The experimental reaction stress was fit to equation Error! Reference source not found. in order to find the material properties.

In all cases, the coefficients of determinations $\mathrm{R}^{2}$ were calculated to determine the goodness of the fits. Also, One way ANOVA was used to quantify the effect of corneal thickness on biomechanical properties; statistical significance of $p<0.05$ was used in all statistical analyses. Data is reported as mean \pm standard deviation.

\section{RESULTS}

Figure 1a shows the stress-strain behavior of corneal samples with seven different thicknesses. The corneal strips with lower hydration showed a stiffer response. Both exponential and power-law expression successfully curve-fitted the stress-strain curves of samples with thickness $\mathrm{t}>0.35 \mathrm{~mm}$. Dashed lines in Figure 1a show the power-law fits. The average tangent modulus for different thickness groups is obtained from the stress-strain curves, Figure $1 \mathrm{~b}$. This plot show that there is a significant difference between tangent modulus of different groups $(\mathrm{P}<0.05)$.

(a)

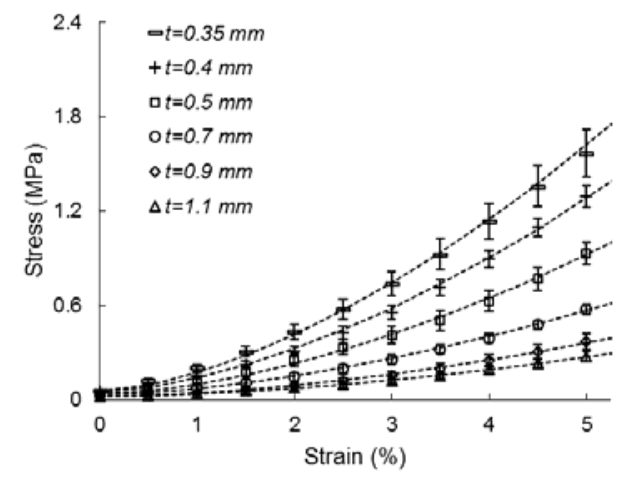

(b)

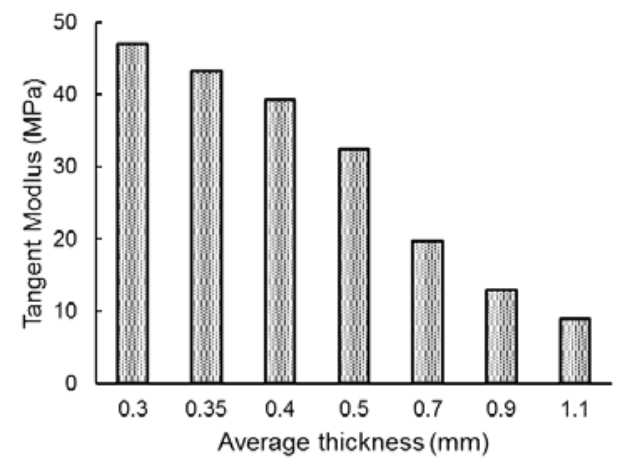

Figure 1: (a) The thickness effects on tensile behavior of corneal strips. The dashed lines show the fits obtained using the power-law relation. (b) The effect of thickness on tangent modulus at 5\% strain.

Figure 2 shows the reaction stress variation as a function of time in a typical stepwise unconfined compression experiment. The reaction stress increased non-linearly during the ramp loading and decreased to an equilibrium stress during the relaxation stage. The slope of the equilibrium stress-strain curve was used to calculate the equilibrium moduli of corneal samples as a function of average thickness, Figures $2 \mathrm{~b}$. ANOVA analysis showed that variation of these moduli was not statistically significant when the thickness is between $1.07 \mathrm{~mm}$ and 1.27 $\mathrm{mm}$. The in-plane Young's modulus and permeability coefficient were determined by fitting the transversely isotropic biphasic model to the experimental measurements. An average coefficient of determination $r^{2}=0.979$ was obtained for the fits. Figures 2c-d show the corneal inplane Young's modulus and permeability coefficient as a function of average thickness. 

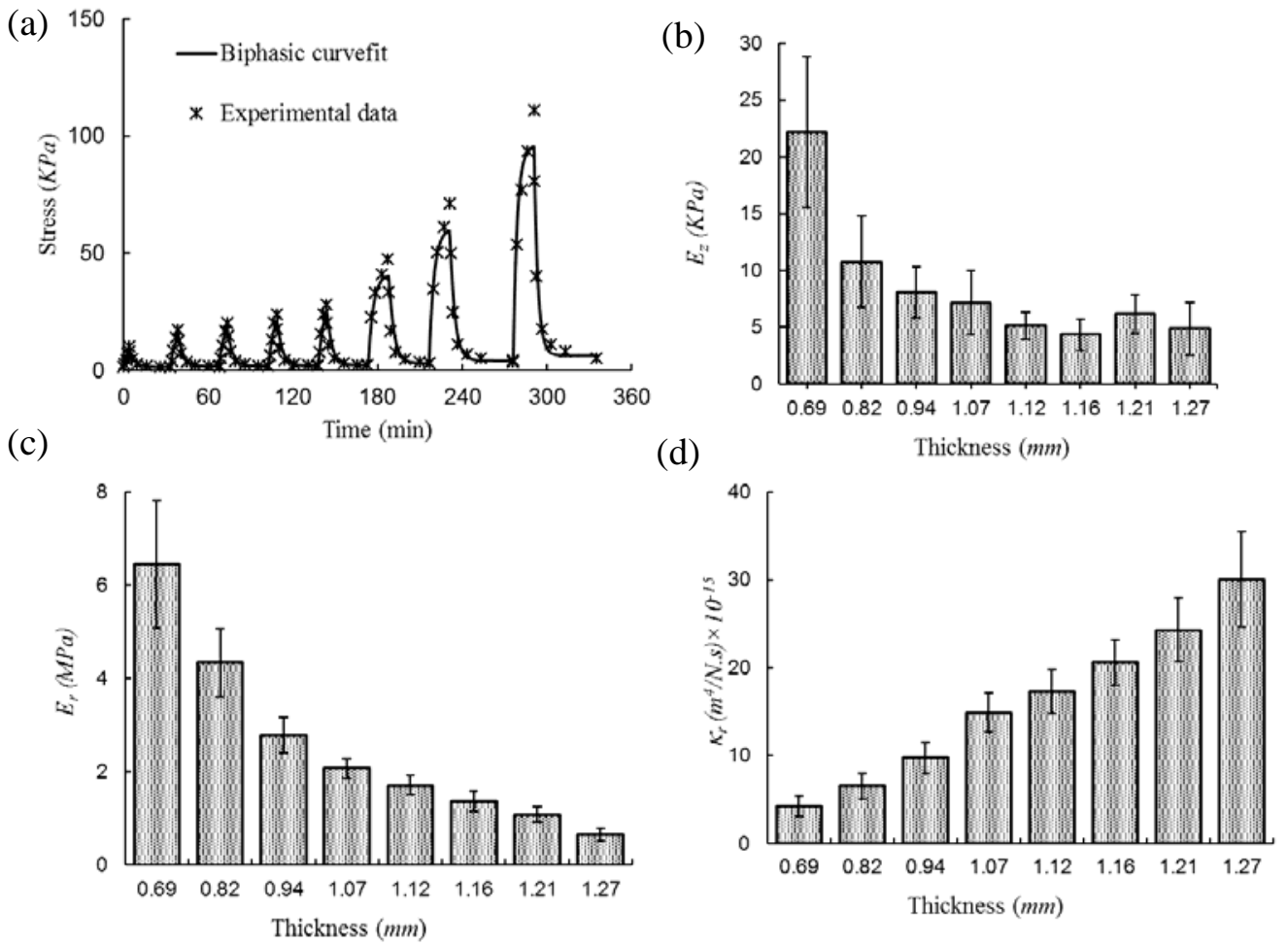

Figure 2: (a) Typical experimental measurements from stepwise unconfined compression tests. The solid line shows the curve-fit of the experimental measurements with a transversely isotropic biphasic model. (b) The equilibrium out-of-plane Young's modulus $\left(E_{z}\right)$, (c) the in-plane Young's modulus, and (d) the permeability coefficient of the cornea as a function of average thickness.

\section{DISCUSSION}

The present study investigated the relation between corneal thickness (hydration) and material properties. For this purpose, the effects of corneal hydration were investigated in extensometry and unconfined compression experiments. This extensometry has been widely used to determine the stress-strain behavior of the cornea; which resulted in a wide range of variations for material parameters [8, 9, 24]. The present study showed that lack of control on thickness (hydration) has significantly contributed to this variation. In order to further investigate the hydration dependent mechanical behavior of the cornea, corneal buttons were tested under unconfined compression. A transversely isotropic model was used to curve-fit the experimental data and obtain the hydration dependent in-plane Young's modulus, out-of-plane compressive modulus, and permeability coefficient of the cornea. It is seen that mechanical properties strongly depend on the corneal thickness (hydration), Figure 2.

The hydration dependent properties of the cornea can be explained in terms of its microstructure. The extracellular matrix of the cornea like many other soft tissues is primarily composed of collagen fibrils and proteoglycans. In particular, regularly distributed collagen fibrils are surrounded by a proteoglycan matrix. The presence of proteoglycan creates a strong tendency for the tissue to swell when immersed in a water-based solution. With increasing thickness (caused by hydration changes), the bonds between collagen fibrils and proteoglycan side chains possibly break and a softer mechanical response is obtained [26-28]. Similarly, with increasing the thickness, the total fixed charge density (and subsequently the osmotic pressure) 
decreases. Therefore, it can be proposed that hydration-dependent properties of the cornea are due to the molecular-level interactions between collagen fibrils and proteoglycans.

\section{CONCLUSIONS}

The present work investigated the hydration dependent material properties of the cornea. Two different experimental techniques, i.e. uniaxial tensile tests and unconfined compression experiments, were used to characterize the corneal mechanical behavior. Furthermore, numerical models were used to analyze the experimental measurements. It was concluded that swollen corneal samples have softer mechanical properties in comparison with dehydrated ones. Therefore, careful attention must be taken in interpreting experimental studies that do not fully control the thickness.

\section{REFERENCES}

[1] D.A. Hoeltzel, et al., Strip Extensiometry for Comparison of the Mechanical Response of Bovine, Rabbit, and Human Corneas. Journal of Biomechanical Engineering. 114(2), 202-215, 1992.

[2] J. Kampmeier, et al., Thermal and Biomechanical Parameters of Porcine Cornea. Cornea. 19(3), 355-363, 2000.

[3] Y. Zeng, et al., A comparison of biomechanical properties between human and porcine cornea. Journal of Biomechanics. 34(4), 533-537, 2001.

[4] G. Wollensak, E. Spoerl, T. Seiler, Stress-strain measurements of human and porcine corneas after riboflavin-ultraviolet-A-induced cross-linking. Journal of Cataract and Refractive Surgery. 29(9), 1780-1785, 2003.

[5] A. Elsheikh, D. Alhasso, Mechanical anisotropy of porcine cornea and correlation with stromal microstructure. Experimental Eye Research. 88(6), 1084-1091, 2009.

[6] B.L. Boyce, et al., Stress-controlled viscoelastic tensile response of bovine cornea. Journal of Biomechanics. 40(11), 2367-2376, 2007.

[7] A. Elsheikh, W. Kassem, S.W. Jones, Strain-rate sensitivity of porcine and ovine corneas. Acta of Bioengineering and Biomechanics. 13(2), 25-36, 2011.

[8] F. Boschetti, et al., Mechanical Characterization of Porcine Corneas. Journal of Biomechanical Engineering. 134(3), 031003-9, 2012.

[9] A. Elsheikh, K. Anderson, Comparative study of corneal strip extensometry and inflation tests. Journal of The Royal Society Interface. 2(3), 177-185, 2005.

[10] A. Elsheikh, D. Alhasso, P. Rama, Biomechanical properties of human and porcine corneas. Experimental Eye Research. 86(5), 783-790, 2008.

[11] B.L. Boyce, et al., Full-field deformation of bovine cornea under constrained inflation conditions. Biomaterials. 29(28), 3896-3904, 2008.

[12] A. Elsheikh, D. Wang, D. Pye, Determination of the modulus of elasticity of the human cornea. Journal of Refractive Surgery. 23(8), 808-18, 2007.

[13] T. Olsen, S. Sperling, The swelling pressure of the human corneal stroma as determined by a new method. Experimental Eye Research. 44(4), 481-490, 1987.

[14] B.O. Hedbys, C.H. Dohlman, A new method for the determination of the swelling pressure of the corneal stroma in vitro. Experimental Eye Research. 2(2), 122-129, 1963. 
[15] M.H. Friedman, et al., Contribution of the Donnan osmotic pressure to the swelling pressure of corneal stroma. American Journal of Physiology. 222(6), 1565-1570, 1972.

[16] T. Wiley, I. Fatt, Effects of solutes on the swelling pressure of the corneal stroma. Investigative Ophthalmology. 14(9), 684-688, 1975.

[17] S. Hodson, et al., Observation on the human cornea in vitro. Exp Eye Res. 32(3), 35360, 1981.

[18] H. Hatami-Marbini, E. Etebu, Rate Dependent Biomechanical Properties of Corneal Stroma in Unconfined Compression Biorheology. 50(3-4), 133-47, 2013.

[19] H. Hatami-Marbini, E. Etebu, An experimental and theoretical analysis of unconfined compression of corneal stroma. Journal of Biomechanics. 46(10), 1752-1758, 2013.

[20] H. Hatami-Marbini, E. Etebu, Hydration dependent biomechanical properties of the corneal stroma. Exp Eye Res. 116, 47-54, 2013.

[21] H. Hatami-Marbini, Hydration Dependent Viscoelastic Tensile Behavior of Cornea. Ann Biomed Eng. 42(8), 1740-8, 2014.

[22] H. Hatami-Marbini, R. Maulik, A Biphasic Transversely Isotropic Poroviscoelastic Model for the Unconfined Compression of Hydrated Soft Tissue. Journal of biomechanical engineering. 138(3), 031003-1-6, 2016.

[23] B.O. Hedbys, S. Mishima, The thickness-hydration relationship of the cornea. Experimental Eye Research. 5(3), 221-228, 1966.

[24] S.L. Woo, et al., Nonlinear material properties of intact cornea and sclera. Exp Eye Res. 14(1), 29-39, 1972.

[25] B. Cohen, W.M. Lai, V.C. Mow, A transversely isotropic biphasic model for unconfined compression of growth plate and chondroepiphysis. Journal of biomechanical engineering. 120(4), 491-6, 1998.

[26] J.E. Scott, Proteoglycan: collagen interactions and corneal ultrastructure. Biochem. Soc. Trans. 19(4), 877-881, 1991.

[27] X. Cheng, H. Hatami-Marbini, P.M. Pinsky, Modeling Collagen-Proteoglycan Structural Interactions in the Human Cornea, in Computer Models in Biomechanics, G.A. Holzapfel and E. Kuhl, Editors., Springer.11-24, 2013.

[28] H. Hatami-Marbini, P.M. Pinsky. On Mechanics of Connective Tissue: Assessing the Electrostatic Contribution to Corneal Stroma Elasticity. in Material Reseaech Society Proceedings. Boston, 2009 